\title{
Ion chromatography of major anions in the Neeru stream, Bhaderwah, J\&K, India
}

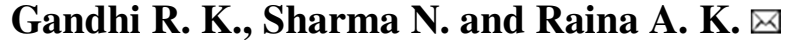

Received: 05.04.2017

Revised: 21.05.2017

Accepted: 28.06.2017

\begin{abstract}
Water samples from 25 different stations along the Neeru stream and its tributaries / sub-tributaries has been collected and analysed for anions viz., Fluoride, Chloride, Nitrate, Phosphate and Sulphate for two years (Jan 2014- Dec 2015) by using Ion chromatography with $3.2 \mathrm{mM}$ sodium carbonate and $1 \mathrm{mM}$ sodium bicarbonate as mobile phase and $50 \mathrm{mM}$ solution of sulphuric acid as suppressor. The observed values of Fluoride, Chloride, Nitrate, Phosphate and Sulphate were found to be in the range of $\mathrm{BDL}-\mathbf{0 . 8 5}, 0.11-9.88,0.02-7.78$, BDL -0.39 and $0.26-9.56$, respectively. Possible reasons for the fluctuations of these anions in the Neeru stream have also been discussed. All analysed anions have been found within WHO and BIS permissible limits.
\end{abstract}

Key words: Anions, Bhaderwah, Ion chromatography, Neeru stream, Water quality

\section{Introduction}

The chemical constitution and properties of surface water in a particular region is determined by natural processes such as precipitation rate, weathering processes, soil erosion and anthropogenic disturbances. However these may be polluted from point and non point sources such as urban, industrial and agricultural activities (Jarvie et al., 1998; Giridharan et al., 2009; Nouri et al., 2009 \& 2011; Garizi et al., 2011). Quality of surface water, both lotic as well as lentic, has been continuously altered by various pollutants contributed by different anthropogenic activities (Varekar et al., 2015). Lotic water bodies, mostly originating from snow covered mountains and constituting a continuous source of water supply to the people living in mountainous watershed as well as downstream, play a significant role in moderating or dispersing municipal, industrial and agricultural waste waters in a watershed (Wang et al., 2007), however, in doing so these water bodies get polluted with different type of pollutants which makes their water unfit for drinking and other domestic purposes. Several studies has been

\footnotetext{
Author's Address

${ }^{1 \& 3}$ Deptt of Environmental Sciences, University of Jammu, Jammu

${ }^{2}$ Institute of Mountain Environment, Bhaderwah Campus, University of Jammu

E-mail.: anilkraina@yahoo.com
}

conducted on the water quality of lotic water bodies with respect to anions and other parameters by various workers (Semwal and Jangwan, 2009; Garizi et al., 2011; Das et al., 2014; Dutta, 2014; Majumdar and Dutta, 2014; Baba et al., 2015; Bora and Goswami, 2015; Sharma et al., 2015 a, b \& c; Bourasi et al., 2016; Dhamodharan et al., 2016; Khadse et al., 2016; Paudyal et al., 2016; Chandan et al., 2017; Rameez and Srivastava, 2017; Ebadati, 2017). However, Neeru stream, an important left bank glacial fed perennial tributary of the river Chenab, has not received due attention except for a stray study conducted by Shekhar (1990). The stream passes through various human inhabited places and also joined by various tributaries / subtributaries before its confluence with the River Chenab at Pul Doda. During its course of journey, it receives waste from municipal and agricultural area including biological waste from slaughters houses, which is seriously affecting its water quality. As it is facing impacts of increasing urbanization and other anthropogenic activities, thereby, monitoring of stream water quality becomes essential for present and future water quality management, for which the present study has been carried out.

Study area: Neeru stream is a glacial fed perennial left bank tributary of the river Chenab which originates from Kaplash Kund (4200 m a.s.1.) and 
equally contributed by Ashapati glacier. While draining the Neeru watershed it passes through Bhaderwah town and other densely populated villages and finally joins the river Chenab at Pul Doda (850 $\mathrm{m}$ a.s.l.) in the Bhaderwah tehsil of district Doda of Jammu and Kashmir (Figure 1). There is a considerable variation in altitude in the study area ( $850 \mathrm{~m}$ to $4200 \mathrm{~m}$ a.s.1.) which results in great climatic variation from snow laden mountains peaks in the upper reaches of Kaplash Kund and Ashapati glacier to almost dry subtropical climate at Pul Doda. This great climatic variation in the Neeru catchment also supports a rich diversity and density of both flora and fauna.

Sampling Stations: Water samples from 25 different stations (Table 1) have been collected for 2 years (Jan 2014- Dec 2015) on monthly basis from the main Neeru stream and its tributaries/ subtributaries for monitoring the water quality of the stream with respect to major anions. At the point of joining of a tributary with the main stream (Neeru stream), water samples has been collected from two stations - one located at $200 \mathrm{~m}$ before the confluence of a tributary with the main stream while the other located at $200 \mathrm{~m}$ after the confluence of a tributary with the main stream, to monitor the effect of water quality of that particular micro watershed on the water quality of the main stream. Geo-coordinates and altitude of different monitoring stations has been recorded using Garmin, Montana 680 (GPS). Samples were collected in the morning hours (9:00-11:00 am) in the polypropylene bottles and sealed on the spot for ion chromatographic analysis at IC laboratory in the Department of Environmental Sciences, University of Jammu, Jammu. Owing to the difficult terrain and large distance between stations, monthly sampling on 25 different stations was completed within 2-3 days.

\section{Material and Methods}

Chemicals and Reagents

Mobile Phase: Mobile phase was prepared using $3.2 \mathrm{mM}$ (milli molar) sodium carbonate and $1 \mathrm{mM}$ sodium bicarbonate in a volumetric flask of $1000 \mathrm{ml}$.

Supressor: $50 \mathrm{mM}$ solution of sulphuric acid was used as suppressor.
Standard: IC Multi-elements standard I for anions $\left(\mathrm{F}^{-}=100 \mathrm{mg} / \mathrm{l}, \mathrm{Cl}^{-}=250 \mathrm{mg} / \mathrm{l}, \mathrm{NO}_{3}{ }^{-}, \mathrm{SO}_{4}{ }^{2-}=500\right.$ $\mathrm{mg} / \mathrm{l}$ and $\left.\mathrm{PO}_{4}{ }^{3-}=1000 \mathrm{mg} / \mathrm{l}\right)$ manufactured by Merck (Millipore) were used for calibration of the IC system.

Ultrapure water: Ultrapure water was prepared using Millipore (DirectQ-3 with pump).

Instrument: Metrohm Ion Chromatograph system (model 850 professional) integrated with Compact Autosampler (model 863) through a computer system has been used for analysis.

\section{Analysis}

a) Sample preparation: Samples were prepared using 1:10 dilution factor (i.e., one $\mathrm{ml}$ of sample was raised to $10 \mathrm{ml}$ using ultrapure water) to avoid ionic saturation of the column.

b) Sample loading: Properly marked sample vials were arranged in the Autosampler for analysis. Information about the sample identification and position was entered in the determination series in the workplace of the MagIC Net software.

c) Sample analysis: Calibration of the system was done using IC Multi-elements standard solution $.0 .1 \mathrm{ml}$ of stock standard solution was raised to $10 \mathrm{ml}$ to get concentrations of $\mathrm{F}^{-}=1$ ppm, $\mathrm{Cl}^{-}=2.5 \mathrm{ppm}, \mathrm{NO}_{3}{ }^{-}, \mathrm{SO}_{4}{ }^{2-}=5 \mathrm{ppm}$ and $\mathrm{PO}_{4}{ }^{3-}=10 \mathrm{ppm}$. Similarly standards of higher concentration were made for calibration of the IC system. Flow rate of $0.7 \mathrm{~mL} / \mathrm{min}$ and Pressure of $12.26 \mathrm{MPa}$ was maintained in the Metrosep A Supp $5-250 / 4.0$ column. $20 \mu 1$ loop was used for sample injection and $30 \mathrm{~min}$ of recording time was set for each sample.

d) Reprocessing of Results: After scheduled recording time of 30 minutes results were obtained in the Database of IC software. The results were reprocessed using $1 \mathrm{ppm}$ standard to phase out any errors in the retention time and percentage window for analyte under consideration.

\section{Results and Discussion}

Average monthly concentration of Fluoride, Chloride, Nitrate, Phosphate and Sulphate for two years (Jan 2014- Dec 2015) in the main stream and tributaries/ sub-tributaries has been presented in the Tables $2 \mathrm{a} \& \mathrm{~b}, 3 \mathrm{a} \& \mathrm{~b}, 4 \mathrm{a} \& \mathrm{~b}, 5 \mathrm{a} \& \mathrm{~b}$ and $6 \mathrm{a} \& \mathrm{~b}$, respectively. 


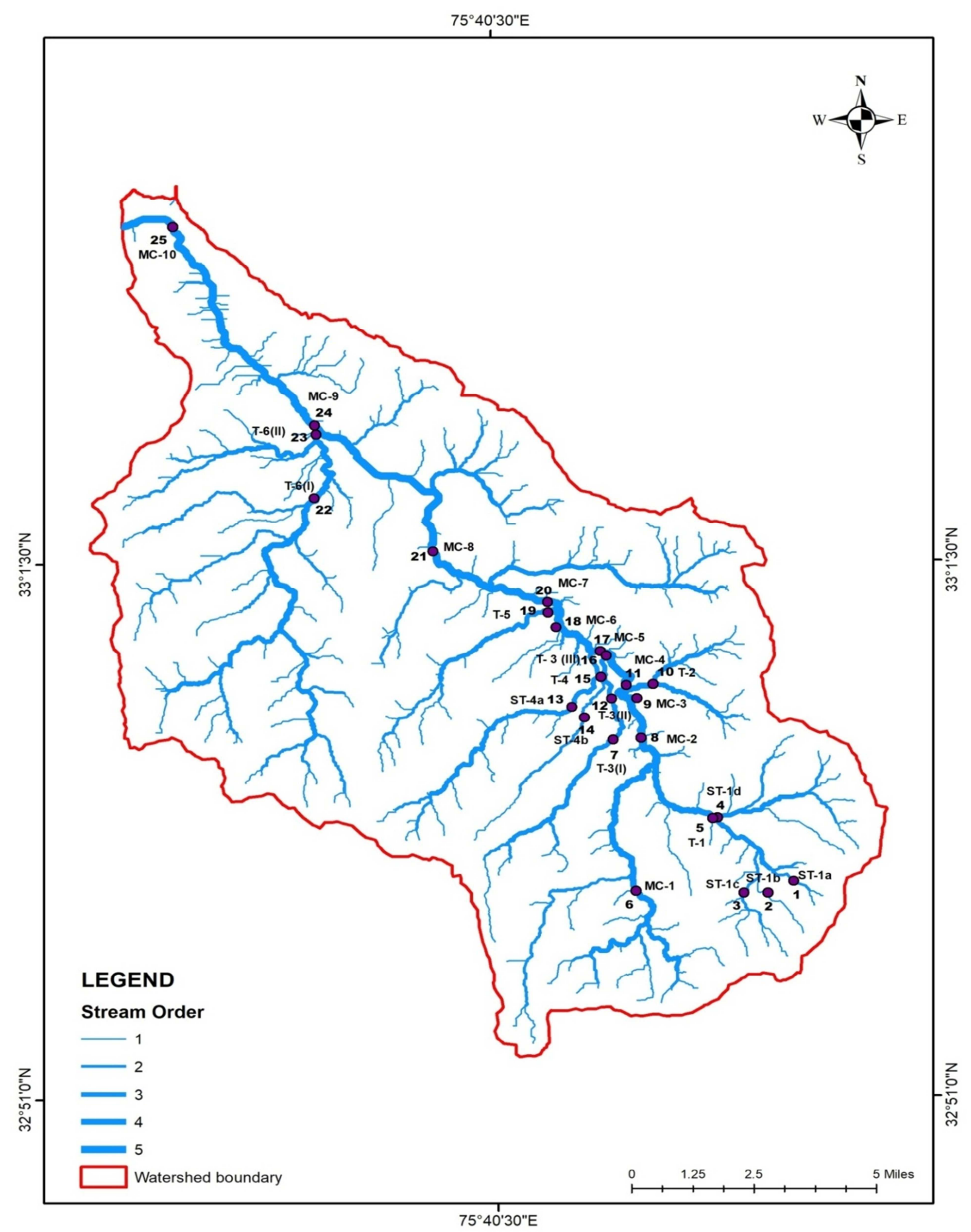

Figure 1: Map showing different sampling stations and stream order in the Neeru watershed. 
Gandhi et al.

Table 1: Details of the different sampling stations at Neeru stream and its tributaries/ sub-tributaries.

\begin{tabular}{|c|c|c|c|c|c|}
\hline S. No. & Station Name & Station Code & Impact Category & Geo-Coordinates & $\begin{array}{l}\text { Altitude (in } \\
\text { meters) }\end{array}$ \\
\hline 1. & Thanalla I & ST-1a & Baseline Station & $32^{0} 55^{\prime} 13.3^{\prime \prime} \mathrm{N} 75^{0} 46^{\prime} 46.6^{\prime \prime} \mathrm{E}$ & 2240 \\
\hline 2. & Thanalla II & ST-1b & Baseline Station & $32^{0} 54^{\prime} 59.8^{\prime \prime} \mathrm{N} 75^{\circ} 46^{\prime} 13.5^{\prime \prime} \mathrm{E}$ & 2184 \\
\hline 3. & Thanalla III & ST-1c & Baseline Station & $32^{0} 54^{\prime} 59.9^{\prime \prime} \mathrm{N} 75^{0} 45^{\prime} 43.4^{\prime \prime} \mathrm{E}$ & 2156 \\
\hline 4. & Bheja I & ST-1d & Trend or Impact Station & $32^{0} 56^{\prime} 28.5^{\prime \prime} \mathrm{N} 75^{\circ} 45^{\prime} 10.5^{\prime \prime} \mathrm{E}$ & 1823 \\
\hline 5. & Bhaja II & T-1 & Trend or Impact Station & $32^{0} 56^{\prime} 27.6^{\prime \prime} \mathrm{N} 75^{\circ} 45^{\prime} 04.7^{\prime \prime} \mathrm{E}$ & 1815 \\
\hline 6. & Thanthera & MC-1 & Baseline Station & $32^{0} 55^{\prime} 03.0^{\prime \prime} \mathrm{N} 75^{\circ} 43^{\prime} 26.5^{\prime \prime} \mathrm{E}$ & 2163 \\
\hline 7. & Puneja & T-3(I) & Trend or Impact Station & $32^{0} 58^{\prime} 01.2^{\prime \prime} \mathrm{N} 75^{\circ} 42^{\prime} 59.4^{\prime \prime} \mathrm{E}$ & 1733 \\
\hline 8. & Dareja & MC-2 & Trend or Impact Station & $32^{0} 58^{\prime} 03.2^{\prime \prime} \mathrm{N} 75^{0} 43^{\prime} 34.4^{\prime \prime} \mathrm{E}$ & 1683 \\
\hline 9. & Gupt Ganga & MC-3 & Trend or Impact Station & $32^{0} 58^{\prime} 49.4^{\prime \prime} \mathrm{N} 75^{\circ} 42^{\prime} 57.4^{\prime \prime} \mathrm{E}$ & 1638 \\
\hline 10. & Atalgarh & T-2 & Trend or Impact Station & $32^{0} 58^{\prime} 39.7^{\prime \prime} \mathrm{N} 75^{\circ} 42^{\prime} 07.1^{\prime \prime} \mathrm{E}$ & 1682 \\
\hline 11. & Renda & MC-4 & Trend or Impact Station & $32^{0} 58^{\prime} 27.5^{\prime \prime} \mathrm{N} 75^{0} 42^{\prime} 22.6^{\prime \prime} \mathrm{E}$ & 1700 \\
\hline 12. & Dharampura & T-3(II) & Trend or Impact Station & $32^{0} 59^{\prime} 15.1^{\prime \prime} \mathrm{N} 75^{\circ} 42^{\prime} 44.0^{\prime \prime} \mathrm{E}$ & 1572 \\
\hline 13. & Launcher Morh & ST-4a & Trend or Impact Station & $32^{0} 58^{\prime} 49.5^{\prime \prime} \mathrm{N} 75^{\circ} 43^{\prime} 29.2^{\prime \prime} \mathrm{E}$ & 1628 \\
\hline 14. & Hallayan & ST-4b & Trend or Impact Station & $32^{0} 59^{\prime} 06.6^{\prime \prime} \mathrm{N} 75^{\circ} 43^{\prime} 50.1^{\prime \prime} \mathrm{E}$ & 1636 \\
\hline 15. & College link Road & T-4 & Trend or Impact Station & $32^{\circ} 59^{\prime} 05.2^{\prime \prime} \mathrm{N} 75^{\circ} 43^{\prime} 16.2^{\prime \prime} \mathrm{E}$ & 1553 \\
\hline 16. & Sarol Bagh I & T-3(III) & Trend or Impact Station & $33^{\circ} 00^{\prime} 13.3^{\prime \prime} \mathrm{N} 75^{\circ} 41^{\prime} 47.6^{\prime \prime} \mathrm{E}$ & 1467 \\
\hline 17. & Sarol Bagh II & MC-5 & Trend or Impact Station & $33^{\circ} 00^{\prime} 43.1^{\prime \prime} \mathrm{N} 75^{\circ} 41^{\prime} 37.2^{\prime \prime} \mathrm{E}$ & 1423 \\
\hline 18. & Domail & MC-6 & Trend or Impact Station & $33^{0} 00^{\prime} 30.8^{\prime \prime} \mathrm{N} 75^{0} 41^{\prime} 37.9^{\prime \prime} \mathrm{E}$ & 1480 \\
\hline 19. & Hanga Nallah & T-5 & Trend or Impact Station & $32^{0} 59^{\prime} 40.3^{\prime \prime} \mathrm{N} 75^{\circ} 42^{\prime} 51.0^{\prime \prime} \mathrm{E}$ & 1521 \\
\hline 20. & Amira Nagar & MC-7 & Trend or Impact Station & $32^{0} 59^{\prime} 45.3^{\prime \prime} \mathrm{N} 75^{\circ} 42^{\prime} 43.2^{\prime \prime} \mathrm{E}$ & 1510 \\
\hline 21. & Dhrudu & MC-8 & Trend or Impact Station & $33^{0} 01^{\prime} 43.4^{\prime \prime} \mathrm{N} 75^{0} 39^{\prime} 12.3^{\prime \prime} \mathrm{E}$ & 1334 \\
\hline 22. & Mallothi & T-6(I) & Trend or Impact Station & $33^{\circ} 02^{\prime} 46.9^{\prime \prime} \mathrm{N} 75^{0} 36^{\prime} 42.3^{\prime \prime} \mathrm{E}$ & 1357 \\
\hline 23. & Bhalla I & T-6(II) & Trend or Impact Station & $33^{\circ} 04^{\prime} 02.3^{\prime \prime} \mathrm{N} 75^{\circ} 36^{\prime} 45.1^{\prime \prime} \mathrm{E}$ & 1202 \\
\hline 24. & Bhalla II & MC-9 & Trend or Impact Station & $33^{\circ} 04^{\prime} 12.9^{\prime \prime} \mathrm{N} 75^{\circ} 36^{\prime} 43.3^{\prime \prime} \mathrm{E}$ & 1185 \\
\hline 25. & Galgander & MC-10 & Trend or Impact Station & $33^{0} 08^{\prime} 07.5^{\prime \prime} \mathrm{N} 75^{0} 33^{\prime} 44.4^{\prime \prime} \mathrm{E}$ & 863 \\
\hline
\end{tabular}

MC= Main Stream, T= Tributary, ST= Sub Tributary

20

Environment Conservation Journal 
Ion chromatography of major anions in the Neeru stream

Table 2a: Average monthly fluoride concentration at different stations along Neeru stream for two years (Jan 2014 to Dec 2015).

\begin{tabular}{|c|c|c|c|c|c|c|c|c|c|c|c|c|}
\hline $\begin{array}{l}\text { Station } \\
\text { Month } \\
\end{array}$ & Jan & Feb & Mar & Apr & May & Jun & Jul & Aug & Sep & Oct & Nov & Dec \\
\hline \multicolumn{13}{|c|}{ Baseline stations } \\
\hline MC-1 (S6) & $\mathrm{BDL}$ & $\mathrm{BDL}$ & $\mathrm{BDL}$ & 0.07 & 0.11 & 0.04 & 0.13 & 0.03 & 0.06 & 0.13 & 0.02 & $\mathrm{BDL}$ \\
\hline \multicolumn{13}{|c|}{ Impact/trend stations } \\
\hline $\mathrm{MC}-2(\mathrm{~S} 8)$ & BDL & $\mathrm{BDL}$ & 0.11 & 0.24 & 0.83 & 0.38 & 0.18 & 0.17 & 0.07 & 0.06 & BDL & $\mathrm{BDL}$ \\
\hline $\mathrm{MC}-3$ (S9) & BDL & 0.06 & 0.03 & 0.19 & 0.48 & 0.19 & 0.14 & 0.10 & 0.06 & 0.05 & $\mathrm{BDL}$ & $\mathrm{BDL}$ \\
\hline MC-4 (S11) & BDL & $\mathrm{BDL}$ & 0.09 & 0.08 & 0.17 & 0.08 & $\mathrm{BDL}$ & $\mathrm{BDL}$ & $\mathrm{BDL}$ & 0.06 & BDL & 0.16 \\
\hline MC-5 (S17) & $\mathrm{BDL}$ & $\mathrm{BDL}$ & 0.04 & 0.09 & 0.21 & 0.12 & 0.10 & 0.10 & 0.09 & $\mathrm{BDL}$ & $\mathrm{BDL}$ & $\mathrm{BDL}$ \\
\hline MC-6 (S18) & BDL & BDL & 0.09 & 0.08 & 0.17 & 0.08 & BDL & BDL & $\mathrm{BDL}$ & 0.06 & $\mathrm{BDL}$ & 0.16 \\
\hline MC-7 (S20) & $\mathrm{BDL}$ & 0.05 & 0.06 & 0.16 & 0.85 & 0.46 & 0.14 & 0.11 & 0.11 & 0.13 & 0.06 & $\mathrm{BDL}$ \\
\hline MC-8 (S21) & $\mathrm{BDL}$ & $\mathrm{BDL}$ & 0.05 & 0.08 & 0.36 & 0.15 & 0.13 & 0.11 & 0.10 & 0.07 & 0.05 & $\mathrm{BDL}$ \\
\hline MC-9 (S24) & BDL & $\mathrm{BDL}$ & 0.09 & 0.13 & 0.14 & 0.15 & 0.12 & 0.10 & 0.09 & 0.07 & 0.03 & $\mathrm{BDL}$ \\
\hline MC-10(S25) & BDL & $\mathrm{BDL}$ & $\mathrm{BDL}$ & 0.11 & 0.18 & 0.16 & 0.12 & 0.11 & 0.09 & 0.05 & $\mathrm{BDL}$ & $\mathrm{BDL}$ \\
\hline
\end{tabular}

Table 2b: Average monthly fluoride concentration at different stations on tributaries/ sub-tributaries for two years (Jan 2014 to Dec 2015).

\begin{tabular}{|l|l|l|l|l|l|l|l|l|l|l|l|l|l|}
\hline $\begin{array}{l}\text { Station } \\
\text { Month }\end{array}$ & Jan & Feb & Mar & Apr & May & Jun & Jul & Aug & Sep & Oct & Nov & Dec \\
\hline \multicolumn{8}{|c|}{ Baseline stations } \\
\hline ST-1a (S1) & BDL & BDL & BDL & 0.07 & 0.07 & 0.14 & 0.39 & 0.10 & 0.16 & 0.09 & 0.06 & BDL \\
\hline ST-1b (S2) & BDL & BDL & BDL & 0.08 & 0.03 & 0.04 & 0.03 & 0.05 & 0.03 & BDL & BDL & BDL \\
\hline ST-1c (S3) & BDL & BDL & BDL & 0.02 & 0.17 & 0.20 & 0.04 & 0.02 & BDL & BDL & BDL & BDL \\
\hline & \multicolumn{10}{|c|}{ Impact/trend stations } & & & \multicolumn{1}{|l|}{} \\
\hline ST-1d (S4) & BDL & BDL & BDL & 0.05 & 0.69 & 0.12 & 0.03 & 0.06 & 0.03 & BDL & BDL & BDL \\
\hline T-1 (S5) & BDL & 0.04 & 0.08 & 0.14 & 0.21 & 0.22 & 0.17 & 0.14 & 0.20 & 0.06 & BDL & BDL \\
\hline T-2 (S10) & BDL & 0.04 & 0.10 & 0.16 & 0.57 & 0.19 & 0.1 & 0.15 & 0.10 & 0.04 & 0.02 & BDL \\
\hline T-3(I) (S7) & 0.06 & 0.02 & 0.12 & 0.3 & 0.62 & 0.15 & 0.05 & 0.07 & 0.08 & 0.13 & 0.09 & BDL \\
\hline T-3(II) (S12) & BDL & 0.05 & 0.17 & 0.21 & 0.76 & 0.21 & 0.15 & 0.06 & 0.13 & 0.09 & BDL & BDL \\
\hline T-3 (III)(S16) & BDL & BDL & 0.08 & 0.09 & 0.19 & 0.11 & 0.09 & 0.07 & 0.04 & BDL & BDL & BDL \\
\hline ST-4a (S13) & BDL & 0.14 & 0.34 & 0.84 & 0.20 & 0.15 & 0.11 & 0.07 & 0.03 & BDL & BDL & BDL \\
\hline ST-4b (S14) & 0.06 & 0.04 & 0.08 & 0.37 & 0.64 & 0.13 & 0.13 & 0.11 & 0.12 & 0.14 & 0.06 & 0.04 \\
\hline T-4 (S15) & BDL & BDL & 0.12 & 0.17 & 0.31 & 0.30 & 0.15 & 0.16 & 0.10 & 0.06 & 0.03 & BDL \\
\hline T-5 (S19) & BDL & 0.04 & 0.07 & 0.13 & 0.21 & 0.20 & 0.13 & 0.12 & 0.07 & 0.06 & BDL & BDL \\
\hline T-6(I) (S22) & BDL & BDL & 0.06 & 0.13 & 0.20 & 0.18 & 0.17 & 0.12 & 0.11 & 0.09 & 0.03 & BDL \\
\hline T-6(II) (S23) & BDL & 0.05 & 0.09 & 0.12 & 0.16 & 0.14 & 0.13 & 0.13 & 0.12 & 0.10 & 0.09 & 0.03 \\
\hline
\end{tabular}

BDL- Below Detection Limits 


\section{Gandhi et al.}

Table 3a: Average monthly chloride concentration at different stations along Neeru stream for two years (Jan 2014 to Dec 2015).

\begin{tabular}{|c|c|c|c|c|c|c|c|c|c|c|c|c|}
\hline Station / Month & Jan & Feb & Mar & Apr & May & Jun & Jul & Aug & Sep & Oct & Nov & Dec \\
\hline \multicolumn{13}{|c|}{ Baseline stations } \\
\hline MC-1 (S6) & 1.49 & 1.34 & 1.22 & 0.67 & 0.48 & 0.51 & 0.54 & 0.81 & 0.92 & 0.97 & 1.15 & 1.37 \\
\hline \multicolumn{13}{|c|}{ Impact/trend stations } \\
\hline $\mathrm{MC}-2$ (S8) & 2.29 & 1.87 & 1.33 & 1.08 & 0.80 & 1.00 & 1.11 & 1.23 & 1.64 & 1.81 & 1.91 & 2.21 \\
\hline $\mathrm{MC}-3$ (S9) & 2.93 & 2.35 & 1.71 & 1.46 & 1.23 & 1.54 & 1.79 & 1.91 & 2.14 & 2.28 & 2.38 & 2.89 \\
\hline MC-4 (S11) & 3.44 & 3.05 & 2.64 & 1.36 & 1.07 & 1.27 & 1.55 & 1.96 & 2.21 & 2.44 & 2.62 & 3.15 \\
\hline MC-5 (S17) & 5.65 & 4.35 & 2.27 & 1.64 & 1.32 & 1.78 & 2.29 & 3.66 & 3.96 & 4.37 & 5.37 & 6.19 \\
\hline MC-6 (S18) & 3.74 & 3.05 & 2.54 & 1.72 & 1.36 & 1.47 & 1.55 & 1.96 & 2.11 & 2.24 & 2.42 & 3.35 \\
\hline MC-7 (S20) & 4.75 & 3.95 & 3.02 & 1.35 & 1.04 & 1.38 & 2.17 & 2.78 & 3.17 & 3.54 & 3.81 & 4.56 \\
\hline MC-8 (S21) & 4.99 & 3.54 & 3.14 & 1.88 & 1.53 & 1.74 & 2.33 & 3.31 & 4.35 & 4.55 & 5.39 & 5.45 \\
\hline MC-9 (S24) & 4.01 & 4.26 & 3.73 & 1.57 & 1.21 & 1.48 & 2.65 & 3.32 & 4.05 & 4.17 & 4.28 & 4.59 \\
\hline $\mathrm{MC}-10(\mathrm{~S} 25)$ & 5.45 & 4.53 & 4.11 & 2.13 & 1.23 & 1.77 & 2.32 & 3.28 & 4.34 & 4.66 & 4.84 & 5.06 \\
\hline
\end{tabular}

Table 3b: Average monthly chloride concentration at different stations on tributaries/ sub-tributaries for two years (Jan 2014 to Dec 2015).

\begin{tabular}{|c|c|c|c|c|c|c|c|c|c|c|c|c|}
\hline Station / Month & Jan & Feb & Mar & Apr & May & Jun & Jul & Aug & Sep & Oct & Nov & Dec \\
\hline \multicolumn{13}{|c|}{ Baseline stations } \\
\hline ST-1a (S1) & 1.65 & 1.46 & 1.37 & 0.73 & 0.35 & 0.27 & 0.21 & 0.98 & 1.19 & 1.29 & 1.45 & 1.61 \\
\hline ST-1b (S2) & 0.75 & 0.54 & 0.43 & 0.19 & 0.11 & 0.21 & 0.26 & 0.37 & 0.58 & 0.61 & 0.73 & 0.76 \\
\hline ST-1c (S3) & 1.25 & 1.13 & 0.91 & 0.35 & 0.31 & 0.47 & 0.61 & 0.64 & 0.69 & 0.73 & 0.9 & 1.16 \\
\hline \multicolumn{13}{|c|}{ Impact/trend stations } \\
\hline ST-1d (S4) & 2.45 & 2.13 & 1.86 & 0.83 & 0.6 & 0.77 & 0.97 & 1.02 & 1.49 & 1.95 & 2.19 & 2.55 \\
\hline $\mathrm{T}-1$ (S5) & 2.06 & 1.67 & 1.52 & 0.94 & 0.72 & 0.85 & 1.01 & 1.24 & 1.29 & 1.37 & 1.93 & 2.04 \\
\hline $\mathrm{T}-2(\mathrm{~S} 10)$ & 3.61 & 3.16 & 2.99 & 1.57 & 1.83 & 2.07 & 2.51 & 2.54 & 2.75 & 2.83 & 3.07 & 3.71 \\
\hline $\mathrm{T}-3(\mathrm{I})(\mathrm{S} 7)$ & 2.82 & 2.74 & 2.57 & 1.35 & 0.82 & 1.13 & 1.55 & 2.04 & 2.23 & 2.32 & 2.52 & 2.68 \\
\hline T-3(II) (S12) & 9.08 & 9.31 & 7.58 & 2.11 & 1.70 & 2.25 & 3.66 & 4.32 & 5.64 & 6.41 & 7.43 & 9.59 \\
\hline T- 3 (III) (S16) & 9.18 & 9.11 & 7.6 & 2.65 & 2.42 & 3.33 & 3.87 & 7.54 & 9.4 & 9.08 & 9.88 & 8.95 \\
\hline ST-4a (S13) & 1.36 & 1.09 & 0.84 & 0.65 & 0.55 & 0.59 & 0.78 & 0.94 & 1.02 & 1.14 & 1.28 & 1.44 \\
\hline ST-4b (S14) & 2.99 & 2.14 & 1.67 & 1.24 & 1.42 & 1.65 & 2.23 & 2.50 & 2.87 & 2.75 & 2.5 & 2.67 \\
\hline $\mathrm{T}-4$ (S15) & 7.41 & 7.33 & 6.88 & 2.76 & 1.83 & 2.44 & 4.43 & 4.94 & 6.42 & 6.97 & 7.93 & 7.70 \\
\hline $\mathrm{T}-5$ (S19) & 4.34 & 3.31 & 2.59 & 1.33 & 1.13 & 1.41 & 1.89 & 2.21 & 2.32 & 3.02 & 3.18 & 3.63 \\
\hline T-6(I) (S22) & 2.93 & 2.31 & 1.89 & 1.22 & 0.95 & 1.21 & 1.83 & 1.98 & 2.42 & 2.43 & 2.23 & 2.68 \\
\hline T-6(II) (S23) & 3.12 & 2.55 & 2.12 & 1.47 & 1.18 & 1.43 & 2.44 & 2.72 & 2.53 & 2.47 & 3.01 & 3.08 \\
\hline
\end{tabular}


Ion chromatography of major anions in the Neeru stream

Table 4a: Average monthly nitrate concentration at different stations along Neeru stream for two years (Jan 2014 to Dec 2015).

\begin{tabular}{|c|c|c|c|c|c|c|c|c|c|c|c|c|}
\hline Station / Month & Jan & Feb & Mar & Apr & May & Jun & Jul & Aug & Sep & Oct & Nov & Dec \\
\hline \multicolumn{13}{|c|}{ Baseline stations } \\
\hline MC-1 (S6) & 0.60 & 1.25 & 1.32 & 5.10 & 2.87 & 2.42 & 1.99 & 1.81 & 1.41 & 1.09 & 0.84 & 0.56 \\
\hline \multicolumn{13}{|c|}{ Impact/trend stations } \\
\hline MC-2 (S8) & 0.77 & 1.49 & 1.73 & 1.88 & 4.18 & 3.69 & 1.37 & 1.25 & 0.85 & 0.18 & 0.08 & 0.04 \\
\hline MC-3 (S9) & 1.67 & 2.17 & 2.69 & 2.95 & 5.65 & 2.67 & 2.25 & 1.66 & 1.38 & 0.47 & 0.31 & 0.28 \\
\hline MC-4 (S11) & 0.05 & 0.94 & 2.79 & 3.70 & 5.07 & 3.31 & 1.46 & 1.42 & 1.35 & 0.7 & 0.4 & 0.36 \\
\hline MC-5 (S17) & 0.44 & 0.62 & 1.66 & 3.54 & 3.6 & 1.22 & 1.16 & 0.9 & 0.89 & 0.67 & 0.48 & 0.22 \\
\hline MC-6 (S18) & 0.05 & 0.94 & 2.79 & 3.70 & 5.07 & 3.31 & 1.46 & 1.42 & 1.35 & 0.7 & 0.4 & 0.36 \\
\hline MC-7 (S20) & 0.64 & 0.78 & 2.04 & 4.05 & 7.33 & 6.68 & 3.55 & 3.48 & 3.19 & 1.97 & 1.4 & 0.78 \\
\hline MC-8 (S21) & 0.03 & 0.76 & 1.33 & 1.92 & 2.69 & 1.61 & 1.19 & 0.17 & 0.14 & 0.11 & 0.07 & 0.06 \\
\hline MC-9 (S24) & 1.13 & 1.28 & 4.34 & 6.59 & 7.01 & 5.42 & 2.27 & 2.1 & 1.95 & 1.13 & 0.78 & 0.70 \\
\hline MC-10 (S25) & 0.53 & 1.04 & 1.50 & 2.42 & 3.96 & 3.58 & 1.28 & 0.96 & 0.79 & 0.69 & 0.48 & 0.39 \\
\hline
\end{tabular}

Table 4b: Average monthly nitrate concentration at different stations on tributaries/ sub-tributaries two years (Jan 2014 to Dec 2015).

\begin{tabular}{|l|l|l|l|l|l|l|l|l|l|l|l|l|l|l|}
\hline Station / Month & Jan & Feb & Mar & Apr & May & Jun & Jul & Aug & Sep & Oct & Nov & Dec \\
\hline \multicolumn{10}{|c|}{ Baseline stations } \\
\hline ST-1a (S1) & 1.86 & 2.35 & 2.60 & 2.85 & 3.46 & 5.88 & 5.47 & 6.1 & 1.66 & 2.00 & 1.45 & 2.24 \\
\hline ST-1b (S2) & 1.98 & 2.41 & 2.63 & 4.01 & 2.22 & 3.35 & 2.09 & 2.00 & 1.76 & 1.40 & 1.32 & 0.85 \\
\hline ST-1c (S3) & 1.99 & 3.02 & 3.53 & 3.53 & 2.82 & 3.03 & 2.21 & 1.85 & 1.54 & 1.38 & 1.30 & 1.17 \\
\hline \multicolumn{8}{|c|}{ Impact/trend stations } \\
\hline ST-1d (S4) & 0.20 & 1.46 & 2.09 & 2.72 & 1.88 & 3.72 & 2.21 & 1.23 & 1.18 & 1.28 & 1.41 & 1.26 \\
\hline T-1 (S5) & 1.52 & 2.56 & 3.08 & 3.65 & 4.28 & 0.35 & 0.41 & 0.3 & 0.22 & 0.68 & 0.95 & 1.22 \\
\hline T-2 (S10) & 1.60 & 1.74 & 1.81 & 1.88 & 5.45 & 3.94 & 1.67 & 1.64 & 0.56 & 0.51 & 0.38 & 0.18 \\
\hline T-3(I) (S7) & 0.02 & 0.13 & 2.10 & 1.84 & 1.75 & 1.46 & 1.34 & 1.25 & 0.81 & 0.13 & 0.12 & 0.10 \\
\hline T-3(II) (S12) & 7.78 & 6.32 & 6.02 & 4.43 & 2.40 & 2.80 & 3.36 & 3.83 & 4.50 & 6.10 & 6.15 & 7.10 \\
\hline T-3 (III)(S16) & 6.66 & 5.39 & 4.10 & 3.21 & 2.73 & 3.23 & 4.53 & 4.45 & 5.05 & 6.06 & 6.61 & 7.56 \\
\hline ST-4a (S13) & 0.73 & 1.38 & 4.07 & 3.22 & 1.44 & 2.39 & 0.57 & 1.14 & 0.93 & 0.71 & 0.15 & 0.10 \\
\hline ST-4b (S14) & 0.57 & 0.80 & 1.72 & 1.91 & 2.02 & 1.81 & 1.77 & 1.12 & 0.55 & 0.31 & 0.28 & 0.20 \\
\hline T-4 (S15) & 7.4 & 6.15 & 4.66 & 3.90 & 3.15 & 4.12 & 4.40 & 5.07 & 5.21 & 6.06 & 6.39 & 7.56 \\
\hline T-5 (S19) & 0.59 & 0.73 & 2.14 & 2.51 & 2.91 & 1.95 & 0.73 & 0.48 & 0.27 & 0.15 & 0.13 & 0.11 \\
\hline T-6(I) (S22) & 0.13 & 0.20 & 1.68 & 1.88 & 3.46 & 3.41 & 1.76 & 1.43 & 0.48 & 0.13 & 0.05 & 0.05 \\
\hline T-6(II) (S23) & 0.71 & 1.30 & 1.39 & 2.44 & 2.52 & 2.07 & 0.99 & 0.72 & 0.60 & 0.56 & 0.40 & 0.29 \\
\hline
\end{tabular}


Gandhi et al.

Table 5a: Average monthly phosphate concentration at different stations along Neeru stream for two years (Jan 2014 to Dec 2015).

\begin{tabular}{|l|l|l|l|l|l|l|l|l|l|l|l|l|l|l|}
\hline $\begin{array}{l}\text { Station } \\
\text { Month }\end{array}$ & Jan & Feb & Mar & Apr & May & Jun & Jul & Aug & Sep & Oct & Nov & Dec \\
\hline \multicolumn{8}{|c|}{ Baseline stations } \\
\hline MC-1 (S6) & BDL & BDL & BDL & BDL & 0.05 & 0.24 & 0.19 & 0.09 & BDL & BDL & BDL & BD \\
\hline \multicolumn{8}{|c|}{ Impact/trend stations } \\
\hline MC-2 (S8) & BDL & BDL & BDL & BDL & BDL & BDL & BDL & BDL & BDL & BDL & BDL & BDL \\
\hline MC-3 (S9) & 0.02 & 0.05 & 0.07 & 0.13 & 0.15 & 0.12 & 0.11 & 0.1 & 0.1 & 0.09 & 0.08 & 0.04 \\
\hline MC-4 (S11) & 0.02 & 0.05 & 0.07 & 0.12 & 0.17 & 0.11 & 0.10 & 0.09 & 0.07 & 0.06 & 0.05 & 0.03 \\
\hline MC-5 (S17) & BDL & BDL & BDL & 0.05 & 0.13 & 0.04 & BDL & BDL & BD & BDL & BDL & BDL \\
\hline MC-6 (S18) & 0.02 & 0.05 & 0.07 & 0.12 & 0.17 & 0.11 & 0.1 & 0.09 & 0.07 & 0.06 & 0.05 & 0.03 \\
\hline MC-7 (S20) & 0.06 & 0.09 & 0.12 & 0.17 & 0.25 & 0.17 & 0.15 & 0.12 & 0.08 & 0.07 & 0.06 & 0.05 \\
\hline MC-8 (S21) & BDL & BDL & BDL & 0.04 & 0.09 & 0.08 & 0.03 & BDL & BDL & BDL & BDL & BDL \\
\hline MC-9 (S24) & BDL & BDL & 0.04 & 0.06 & 0.13 & 0.10 & 0.09 & 0.08 & 0.07 & 0.06 & 0.05 & BDL \\
\hline MC-10 (S25) & BDL & BDL & BDL & BDL & 0.09 & 0.07 & BDL & BDL & BDL & BDL & BDL & BDL \\
\hline
\end{tabular}

Table 5b: Average monthly phosphate concentration at different stations on tributaries/ sub-tributaries for two years (Jan 2014 to Dec 2015).

\begin{tabular}{|c|c|c|c|c|c|c|c|c|c|c|c|c|}
\hline $\begin{array}{l}\text { Station } \\
\text { Month }\end{array}$ & Jan & Feb & Mar & Apr & May & Jun & Jul & Aug & Sep & Oct & Nov & Dec \\
\hline \multicolumn{13}{|c|}{ Baseline stations } \\
\hline ST-1a (S1) & BDL & BDL & $\mathrm{BDL}$ & BDL & $\mathrm{BDL}$ & $\mathrm{BDL}$ & $\mathrm{BDL}$ & BDL & $\mathrm{BDL}$ & BDL & $\mathrm{BDL}$ & $\mathrm{BDL}$ \\
\hline ST-1b (S2) & BDL & BDL & $\mathrm{BDL}$ & BDL & $\mathrm{BDL}$ & $\mathrm{BDL}$ & BDL & $\mathrm{BDL}$ & $\mathrm{BDL}$ & $\mathrm{BDL}$ & $\mathrm{BDL}$ & BDL \\
\hline ST-1c (S3) & BDL & BDL & BDL & BDL & BDL & BDL & BDL & BDL & BDL & BDL & BDL & BDL \\
\hline \multicolumn{13}{|c|}{ Impact/trend stations } \\
\hline ST-1d (S4) & BDL & BDL & $\mathrm{BDL}$ & BDL & $\mathrm{BDL}$ & $\mathrm{BDL}$ & $\mathrm{BDL}$ & $\mathrm{BDL}$ & $\mathrm{BDL}$ & BDL & BDL & BDL \\
\hline T-1 (S5) & $\mathrm{BDL}$ & BDL & $\mathrm{BDL}$ & BDL & $\mathrm{BDL}$ & BDL & $\mathrm{BDL}$ & $\mathrm{BDL}$ & BDL & BDL & $\mathrm{BDL}$ & $\mathrm{BDL}$ \\
\hline $\mathrm{T}-2(\mathrm{~S} 10)$ & 0.02 & 0.06 & 0.12 & 0.18 & 0.39 & 0.17 & 0.16 & 0.15 & 0.11 & 0.07 & 0.04 & 0.03 \\
\hline $\mathrm{T}-3(\mathrm{I})(\mathrm{S} 7)$ & $\mathrm{BDL}$ & $\mathrm{BDL}$ & $\mathrm{BDL}$ & $\mathrm{BDL}$ & $\mathrm{BDL}$ & $\mathrm{BDL}$ & $\mathrm{BDL}$ & $\mathrm{BDL}$ & $\mathrm{BDL}$ & $\mathrm{BDL}$ & $\mathrm{BDL}$ & $\mathrm{BDL}$ \\
\hline T-3(II) (S12) & 0.06 & 0.08 & 0.11 & 0.17 & 0.25 & 0.16 & 0.14 & 0.12 & 0.11 & 0.09 & 0.08 & 0.05 \\
\hline T-3 (III)(S16) & 0.09 & 0.07 & 0.09 & 0.11 & 0.15 & 0.1 & 0.08 & 0.07 & 0.06 & 0.09 & 0.07 & 0.09 \\
\hline ST-4a (S13) & BDL & BDL & 0.05 & 0.15 & 0.17 & 0.11 & 0.05 & BDL & BDL & BDL & BDL & BDL \\
\hline ST-4b (S14) & 0.03 & 0.06 & 0.13 & 0.15 & 0.28 & 0.16 & 0.11 & 0.1 & 0.09 & 0.07 & 0.03 & 0.03 \\
\hline $\mathrm{T}-4$ (S15) & 0.17 & 0.12 & 0.15 & 0.12 & 0.35 & 0.25 & 0.21 & 0.14 & 0.13 & 0.12 & 0.14 & 0.12 \\
\hline $\mathrm{T}-5$ (S19) & $\mathrm{BDL}$ & $\mathrm{BDL}$ & $\mathrm{BDL}$ & 0.09 & 0.12 & 0.06 & $\mathrm{BDL}$ & $\mathrm{BDL}$ & $\mathrm{BDL}$ & $\mathrm{BDL}$ & $\mathrm{BDL}$ & $\mathrm{BDL}$ \\
\hline T-6(I) (S22) & $\mathrm{BDL}$ & $\mathrm{BDL}$ & $\mathrm{BDL}$ & 0.02 & 0.05 & 0.09 & 0.06 & $\mathrm{BDL}$ & $\mathrm{BDL}$ & $\mathrm{BDL}$ & $\mathrm{BDL}$ & BDL \\
\hline T-6(II) (S23) & BDL & BDL & 0.04 & 0.09 & 0.14 & 0.11 & 0.09 & 0.08 & BDL & $\mathrm{BDL}$ & $\mathrm{BDL}$ & BDL \\
\hline
\end{tabular}


Ion chromatography of major anions in the Neeru stream

Table 6a: Average monthly sulphate concentration at different stations along Neeru stream for two years (Jan 2014 to Dec 2015).

\begin{tabular}{|c|c|c|c|c|c|c|c|c|c|c|c|c|}
\hline Station / Month & Jan & Feb & Mar & Apr & May & Jun & Jul & Aug & Sep & Oct & Nov & Dec \\
\hline \multicolumn{13}{|c|}{ Baseline stations } \\
\hline MC-1 (S6) & 3.69 & 1.93 & 1.63 & 1.53 & 0.73 & 1.13 & 2.15 & 2.29 & 2.47 & 2.79 & 2.93 & 3.59 \\
\hline \multicolumn{13}{|c|}{ Impact/trend stations } \\
\hline $\mathrm{MC}-2$ (S8) & 4.48 & 2.07 & 2.03 & 1.12 & 0.85 & 1.25 & 1.64 & 1.88 & 3.39 & 4.02 & 4.23 & 4.72 \\
\hline $\mathrm{MC}-3$ (S9) & 2.95 & 2.12 & 1.79 & 1.76 & 0.84 & 2.17 & 3.38 & 4.78 & 4.22 & 2.24 & 1.91 & 1.46 \\
\hline MC-4 (S11) & 4.59 & 4.51 & 2.88 & 1.83 & 1.28 & 1.91 & 3.48 & 3.85 & 3.84 & 3.24 & 3.43 & 3.80 \\
\hline MC-5 (S17) & 2.93 & 2.64 & 2.47 & 1.58 & 1.39 & 1.86 & 3.06 & 4.08 & 3.62 & 3.46 & 3.21 & 2.65 \\
\hline MC-6 (S18) & 4.59 & 4.51 & 2.88 & 1.83 & 1.28 & 1.91 & 3.48 & 3.85 & 3.84 & 3.24 & 3.43 & 3.80 \\
\hline MC-7 (S20) & 4.91 & 4.35 & 4.09 & 1.94 & 1.27 & 1.67 & 2.8 & 4.19 & 3.84 & 3.03 & 2.87 & 2.39 \\
\hline MC-8 (S21) & 5.57 & 5.23 & 4.18 & 2.28 & 1.18 & 1.75 & 3.91 & 6.00 & 5.33 & 5.07 & 4.96 & 3.80 \\
\hline MC-9 (S24) & 3.86 & 3.17 & 2.87 & 1.87 & 1.74 & 2.26 & 2.65 & 4.08 & 3.51 & 3.46 & 2.88 & 2.71 \\
\hline $\mathrm{MC}-10(\mathrm{~S} 25)$ & 3.78 & 3.05 & 2.77 & 1.94 & 1.34 & 1.54 & 3.26 & 4.66 & 3.74 & 3.57 & 3.5 & 3.89 \\
\hline
\end{tabular}

Table 6b: Average monthly sulphate concentration at different stations on tributaries/ sub-tributaries for two years (Jan 2014 to Dec 2015).

\begin{tabular}{|l|l|l|l|l|l|l|l|l|l|l|l|l|l|l|}
\hline \multicolumn{1}{|c|}{ Station / Month } & Jan & Feb & Mar & Apr & May & Jun & Jul & Aug & Sep & Oct & Nov & Dec \\
\hline \multicolumn{8}{|c|}{ Baseline stations } \\
\hline ST-1a (S1) & 2.79 & 2.34 & 2.12 & 1.89 & 1.33 & 2.09 & 3.23 & 5.27 & 3.37 & 3.68 & 3.5 & 2.37 \\
\hline ST-1b (S2) & 2.17 & 1.71 & 1.48 & 1.09 & 0.9 & 0.26 & 0.68 & 1.14 & 1.9 & 2.63 & 1.66 & 2.02 \\
\hline ST-1c (S3) & 2.37 & 1.81 & 1.53 & 1.13 & 1.14 & 1.08 & 1.14 & 1.57 & 1.83 & 2.39 & 2.55 & 2.68 \\
\hline \multicolumn{7}{|c|}{ Impact/trend stations } \\
\hline ST-1d (S4) & 9.56 & 7.83 & 6.96 & 6.1 & 4.85 & 4.79 & 6.05 & 6.18 & 7.61 & 7.67 & 8.74 & 9.18 \\
\hline T-1 (S5) & 7.38 & 6.04 & 5.36 & 3.33 & 2.06 & 2.66 & 4.01 & 4.17 & 5.86 & 6.65 & 6.53 & 8.64 \\
\hline T-2 (S10) & 4.30 & 4.07 & 3.93 & 2.78 & 2.39 & 2.76 & 3.4 & 4.48 & 3.99 & 3.29 & 3.03 & 3.22 \\
\hline T-3(I) (S7) & 3.07 & 2.34 & 2.14 & 1.55 & 0.78 & 0.82 & 0.97 & 1.73 & 1.98 & 2.1 & 2.46 & 3.02 \\
\hline T-3(II) (S12) & 3.06 & 2.32 & 2.16 & 1.13 & 0.83 & 1.01 & 2.66 & 3.72 & 2.66 & 2.45 & 2.36 & 1.23 \\
\hline T-3 (III)(S16) & 2.04 & 2.58 & 2.19 & 1.72 & 1.11 & 1.33 & 2.37 & 3.36 & 2.81 & 1.84 & 1.82 & 1.66 \\
\hline ST-4a (S13) & 2.83 & 3.51 & 1.87 & 0.86 & 1.45 & 2.18 & 3.45 & 4.37 & 3.17 & 2.81 & 3.01 & 3.17 \\
\hline ST-4b (S14) & 2.61 & 2.09 & 1.89 & 1.73 & 1.29 & 1.65 & 2.62 & 3.69 & 2.99 & 2.67 & 2.59 & 2.46 \\
\hline T-4 (S15) & 2.06 & 2.01 & 1.96 & 1.56 & 1.24 & 1.27 & 1.56 & 2.54 & 2.22 & 2.09 & 1.88 & 1.67 \\
\hline T-5 (S19) & 2.27 & 2.31 & 1.33 & 1.6 & 1.01 & 1.19 & 2.13 & 2.88 & 2.06 & 1.56 & 1.68 & 1.57 \\
\hline T-6(I) (S22) & 1.48 & 1.52 & 1.25 & 1.2 & 0.82 & 0.88 & 1.59 & 1.66 & 1.4 & 1.34 & 1.24 & 1.76 \\
\hline T-6(II) (S23) & 2.66 & 2.13 & 1.64 & 1.35 & 1.15 & 1.18 & 1.7 & 2.96 & 2.82 & 2.73 & 2.7 & 1.98 \\
\hline
\end{tabular}


Gandhi et al.

Table 7: WHO and BIS drinking water acceptable and permissible limits for analysed anions.

\begin{tabular}{|c|c|c|c|c|c|}
\hline S. & Anion Species & \multicolumn{2}{|c|}{ WHO, 1999 } & \multicolumn{2}{c|}{ BIS, 2012 } \\
\cline { 2 - 6 } No. & & Acceptable & Permissible & Acceptable & Permissible \\
\hline 1. & Fluoride $(\mathrm{mg} / \mathrm{l})$ & $*$ & $*$ & 1.0 & 1.5 \\
\hline 2. & Chloride $(\mathrm{mg} / \mathrm{l})$ & 250 & 1000 & 250 & 1000 \\
\hline 3. & Nitrate $(\mathrm{mg} / \mathrm{l})$ & 45 & 100 & 45 & No relaxation \\
\hline 4. & Sulphate $(\mathrm{mg} / \mathrm{l})$ & 200 & 400 & 200 & 400 \\
\hline 5. & Phosphate $(\mathrm{mg} / \mathrm{l})$ & 0.1 & 1.0 & $*$ & $*$ \\
\hline
\end{tabular}

* Limits not provided by the concerned agency.

Table 8a: Correlation analysis of various analysed anions at main stream stations.

\begin{tabular}{|c|c|c|c|c|c|}
\hline Anions & Fluoride & Chloride & Nitrate & Phosphate & Sulphate \\
\hline Fluoride & 1 & & & & \\
\hline Chloride & -0.84 & 1 & & & \\
\hline Nitrate & $0.89^{*}$ & -0.93 & 1 & & \\
\hline Phosphate & $0.93^{*}$ & -0.95 & $0.90^{*}$ & & \\
\hline Sulphate & -0.82 & $0.82^{*}$ & -0.91 & -0.82 & 1 \\
\hline
\end{tabular}

*Statistically significant positive relationships, $\mathrm{p}<0.05$.

Table 8b: Correlation analysis of various analysed anions at stations on tributaries/ sub-tributaries.

\begin{tabular}{|c|c|c|c|c|c|}
\hline Anions & Fluoride & Chloride & Nitrate & Phosphate & Sulphate \\
\hline Fluoride & 1 & & & & \\
\hline Chloride & -0.92 & 1 & & & \\
\hline Nitrate & $0.63^{*}$ & -0.66 & 1 & & \\
\hline Phosphate & $0.98^{*}$ & -0.92 & $0.70^{*}$ & 1 & 1 \\
\hline Sulphate & -0.87 & $0.88^{*}$ & -0.79 & -0.91 & \\
\hline
\end{tabular}

*Statistically significant positive relationships, $\mathrm{p}<0.05$.

Acceptable and permissible limits of various tributaries T-3 I, T-3 II and ST-4a, can be attributed analysed anions as per WHO and BIS have been to the geological contribution by micro-watersheds listed in the Table 7. Correlation between various drained by these tributaries / sub-tributaries. anions at sampling stations located on main stream Karthikeyan et al., (2010) has also attributed and tributaries / sub-tributaries has been presented presence of Fluoride in underground water to in the Tables 8a and 8b, respectively. Perusal of geological deposits and geochemistry of the Tables 2 a \& b revealed that the average monthly location. Along the longitudinal profile of the value of Fluoride, an important parameter to access Neeru stream, fluoride values showed decreasing the water quality for drinking purpose, in main trend from upstream to the downstream areas. stream ranges from a maximum of $0.85 \mathrm{mg} / \mathrm{l}$ in the Though, little bit higher concentration of fluoride month of May (MC-7) to a minimum of BDL at has been detected at some stations (MC-7, T-3 I different stations during different months whereas and ST-4a) it has remained within the permissible in tributaries / sub-tributaries, average monthly limits of $1.5 \mathrm{mg} / \mathrm{l}$ (BIS, 2012) (Table 7) in the fluoride concentration ranged from a minimum Neeru stream. However, Fluoride concentrations value of BDL at different stations during different exceeded the maximum permissible limits of 1.5 months to a maximum of $0.84 \mathrm{mg} / \mathrm{l}$ in the month of $\mathrm{mg} / \mathrm{l}$ WHO (2004) in about $22.7 \%$ of the drinking April (ST-4a). Concentrations of Fluoride present water samples collected from Doda district by in most natural waters are small, generally less than Central Ground Water Board (2010), which results $1.0 \mathrm{mg} / \mathrm{l}$ (Hem, 1985). The comparatively higher in the prevalence of the fluorosis in some of the concentration of fluoride in tributaries / sub-

region of District Doda (Arya et al., 2013). 
Chloride is a major component which maintains the cation-anion balance of ecosystem. Concentration of Chloride ranged between minimum average monthly values of $0.11 \mathrm{mg} / \mathrm{l}$ in the month of May (ST-1b) to a maximum value of $9.88 \mathrm{mg} / \mathrm{l}$ recorded in the month of November (T-3 III). The value of chlorides in the Neeru stream has been observed to be comparatively lower than some of the other Indian rivers where the concentration of chloride has been found to range between $198.57-1200.00$ $\mathrm{mg} / \mathrm{l}$ (Vaishali and Punita, 2013). However, similar lower values of Chloride in the River Bhagirathi, River Torsa and River Teesta and stream Relli has been observed by Gautam (1990), Bhadra et al., (2003) and Acharjee (2013), respectively. Thresh et al., (1949) and Sharma et al., (2015a) suggested Chloride concentration as indication of organic waste primarily of animal origin. In the present investigation, slightly higher values of Chloride in colder periods (October to March) have been recorded at sampling stations (T-3II, T-3III and T4) affected by sewage and municipal waste of the Bhaderwah town. Along the longitudinal profile of the Neeru stream, Chloride did not show much fluctuation. In general, concentration of Chloride remained within the acceptable limits of $250 \mathrm{mg} / \mathrm{l}$ prescribed by WHO (1999) and BIS (2012).

Nitrate and ammonium, the forms of nitrogen, are very important elements in water fertility. The monthly variation in the Nitrate content in main stream and tributaries / sub-tributaries does not exhibit much variation and its value varies between a minimum of $0.02 \mathrm{mg} / \mathrm{l}$ (January) to a maximum of $7.78 \mathrm{mg} / \mathrm{l}$ (January) at different stations (Tables 4 , a \& b). The maximum concentration of ammonium $(100.59 \mathrm{mg} / \mathrm{l})$ has been recorded in the month of May and minimum $(0.19 \mathrm{mg} / \mathrm{l})$ in the month of January. In Snow fed water bodies like Neeru stream, higher concentrations of nitrates / ammonium during the warmer months can be attributed to melting of snow during spring /summer season which brings the nutrient rich water to the stream. The low value of nitrates / ammonium during colder months might be due to absence of melting of snow. Wetzel (2001) reported the higher content of nitrate in snow as compared to rain and also that rise of nitrates during summers is due to melting of snow. Also the higher values of Nitrate / ammonium during warmer months can be attributed to increase in the process of nitrification at higher temperature that convert organic matter to nitrates and nitrites (Kumar and Singh, 2002; Sivakumar and Jaganathan, 2002). The transport of nitrate from the watershed areas with the runoff water during early summers also contribute towards the highest concentrations of nitrate as has also been recorded in the present study during early summer period in which the study area experience the local rains. This view has also been supported by the findings of Jha and Barat (2003) and Acharjee (2013). Along the longitudinal profile of the Neeru stream nitrates / ammonium did not show much fluctuation, however higher concentrations have been recorded in the middle section of the stream where it receives the tributaries draining the inhabited / agricultural areas. The observed values of Nitrate were found to be much below the acceptable limit of 45mg/l (WHO, 1999; BIS, 2012) (Table 7).

The main supply of Phosphate in natural waters comes from the weathering of phosphorus bearing rocks, leaching of soils of the catchment area by rain, cattle dung and night soil (Jhingran, 1982). From the perusal of the Tables $5 \mathrm{a} \& \mathrm{~b}$, it has been observed that Phosphate in main stream / tributaries / sub-tributaries ranged between minimum values of BDL in all baseline stations and some trend or impact stations to a maximum of $0.39 \mathrm{mg} / \mathrm{l}$ in the month of May at T-2 station. Low values of Phosphate have been reported from various Himalayan rivers (Chakraborty, 1998; Dobriyal et al., 1993; Barat and Jha, 2002 and Thapa et al., 2010). The release of Phosphate from sediments might also contribute to the enrichment of Phosphate during early summer. The increased solar radiations also encourage the biological degradation of organic matter and subsequent release of more Phosphate in early summer season (Kumar and Singh, 2002). Weathering of phosphorus bearing rocks, leaching of soils rich in Phosphorus from catchment area by rains also contribute for higher phosphate in the spring seasons. Chakraborty et al., (1959) has also reported higher Phosphate value during spring due to washings received from catchment areas. Low phosphate level during winter may be due to its sedimentation in the form of ferric complexes in the soil, low Calcium level in the water and low water temperature as has also been reported by Seena yya (1971) and Khan and Siddiqui (1974). Along the 
longitudinal profile of the Neeru stream, Phosphate did not show much fluctuation, however higher concentrations have been recorded in the middle section of the stream where it receives the tributaries draining the inhabited /agricultural areas. The amount of Phosphate found in the present study is mostly within the permissible limits prescribed by World Health Organization (1999) i.e., $1 \mathrm{mg} / \mathrm{l}$ (Table 7). Sulphur, an important component in protein metabolism (Pandey et al., 2000), is also ecologically important for the growth of plants as its short supply may inhibit the development of plankton. In the present investigation, the monthly variations in the sulphates contents varied from a minimum of 0.26 $\mathrm{mg} / \mathrm{l}$ in the month of June to a maximum value of $9.56 \mathrm{mg} / \mathrm{l}$ in the month of January (Tables 6, a and b). Similar monthly variation has also been observed by Baurasi et al. (2016) and Patel et al. (2016). Along the longitudinal profile of the river, Sulphates did not show much fluctuation and its concentrations were found to be much below the acceptable limits of $200 \mathrm{mg} / \mathrm{l}$ as prescribed by WHO (1999) and BIS (2012).

Correlation analysis revealed significant positive correlation between Fluoride and Nitrate, $\mathrm{r}(10)=$ 0.89, 0.63; Fluoride and Phosphate, $\mathrm{r}(10)=0.93$, 0.98; Chloride and Sulphate, $\mathrm{r}(10)=0.82,0.88$ and Nitrate and Phosphate, $\mathrm{r}(10)=0.90,0.70$ while significant negative correlation has been observed between Fluoride and Chloride, $\mathrm{r}(10)=-0.84$, 0.92; Fluoride and Sulphate, r $(10)=-0.82,-0.87$; Chloride and Nitrate, $\mathrm{r}(10)=-0.93,-0.66$; Chloride and Phosphate, $r(10)=-0.95,-0.92$; Nitrate and Sulphate, $\mathrm{r}(10)=-0.91,-0.79$ and Phosphate and Sulphate, $\mathrm{r}(10)=-0.82,-0.91$ among all the stations located on the main stream as well as on the tributaries / sub-tributaries at $\mathrm{p}<0.05$, respectively (Table $8 \mathrm{a} \& 8 \mathrm{~b}$ ). Significant positive correlation between pair of anions shows their similar source or chemical process (Sekabira et al., 2010; Gandhi et al., 2017).

\section{Conclusion}

Analysed anions viz., Fluoride, Chloride, Nitrate, Phosphate and Sulphate were found to be within the permissible limits as prescribed by WHO (1999) and BIS (2012). Slightly higher concentrations of Fluoride are contributed by sub-tributaries, ST-4a
\& $4 \mathrm{~b}$, during peak flow months which may be attributed to the increased runoff and geological composition of the micro-watersheds drained by these sub-tributaries. Higher concentrations of Chloride, Nitrate and Phosphate as compared to the rest of the watershed has been observed at stations $\mathrm{T}-3$ (II), T-3(III) and T-4, present in the highly urbanised areas (Bhaderwah town) can be attributed to municipal solid waste, sewage, agricultural runoff and biological waste from slaughter houses which directly or indirectly find their way in these tributaries. Sulphate in higher concentration has been observed at station ST-1d which may be due to the increased deforestation and resulting accelerated soil erosion in the micro-watershed drained by the sub-tributary. Therefore, it can be concluded that rapidly growing population, increased urbanisation and anthropogenic activities in the Neeru watershed are negatively affecting the water quality of the Neeru Stream.

\section{References}

Acharjee, M. L., 2013. Diversity of Plankton and Ichthyofauna in relation to Limnochemistry of River Teesta and Relli in the Darjeeling Himalaya of West Bengal. Ph. D. Thesis submitted to the University of North Bengal, West Bengal, India.

Arya, S., Gazal, S. and Raina, A. K., 2013. Prevalence and severity of dental fluorosis in some endemically afflicted villages of district Doda, Jammu and Kashmir, India. Journal of Applied and Natural Science, 5(2): 406-410.

Baba, D. I., Sharma, K. K. and Shvetambri, 2015. PhysicoChemical Assessment of River Chenab, Jammu And Kashmir. European journal of pharmaceutical and medical research, 2(4): 1048-1064.

Barat, S. and Jha, P., 2002. Changes in the water quality and total coliform bacterial load in a streach of River Mahananda at Siliguri city, West Bengal, India. Asian Journal of Microbiology, Biotechnology and Environmental Sciences, 4(4): 571-575.

Bhadra, B., Mukherjee, S., Chakraborty, R. and Nanda, A. K., 2003. Physico-Chemical and bacteriological investigation on the river Torsa of North Bengal. Journal of Environmental Biology, 24: 125-133.

BIS, 2012. Indian standard drinking water specifications. Bureau of Indian Standards, New Delhi, pp: 1-11.

Bora, M. and Goswami, D. C., 2015. A study on seasonal and temporal variations in physico-chemical and hydrological characteristics of River Kolong at Nagaon Town, Assam, 
India. Archives of Applied Science Research, 7(5): 110117.

Bourasi, S. K., Singh, S. D., Patil, P., Parihar, S. S. and Taunk, A., 2016. Seasonal variation of physico-chemical parameters and diversity of Phytoplankton in river Narmada at Harda (M.P.). CIBTech Journal of Microbology, 5(1): 25-30.

Central Ground Water Board (CGWB), 2010. Ministry of water resources; Ground water quality report of Jammu and Kashmir State. North western Himalayan Region.

Chakraborty, D., 1998. A study on the water and sediment quality as well as macro population of a natural hill stream in the Darjeeling hill. Indian Journal of Environment and Ecoplanning, 1 (3): 69-72.

Chakraborty, R. D., Roy, P. and Singh, S. B., 1959. A quantitative study of the plankton and the physico-chemical condition of the River Jamuna at Allahabad in 1954-55. Indian Journal of Fisheries, 6(1): 186-203.

Chandan, R., Singh, M., Kulkarni, D. and Sohoni, V., 2017. Impact of Industrial Effluents on Physico-Chemical Characteristics of River Basanter Samba (J\&K). International Journal of Innovative Research in Science, Engineering and Technology, 6(3): 3316-3322.

Das, B. K., Boruah, P. and Kar, D., 2014. Study of seasonal variation of water quality of River Siang in Arunachal Pardesh, India. IOSR Journal of Environmental Science, Toxicology and Food Technology, 8(2): 11-20.

Dhamodharan, A., Shanthakumar, S. and Ganapathy, G. P., 2016. Assessment of seasonal variations in surface water quality of Cooum River in Chennai, India - a statistical approach. Global NEST Journal, 18(3): 527-545.

Dobriyal, A. K., Bahuguna, A. K., Kumar, N. and Kotnala, C. B., 1993. Ecology and seasonal diversity of planktons in a spring fed stream Khandagad in Garhwal Himalaya. In Singh, H.R. (Ed.) Advances in Limnology, Narendra Publishing House, New Delhi: 175 -180.

Dutta, S. P. S., 2014. Monthly variations in physico-chemical characteristics of water, MPN index and zooplankton of Devak stream, at Shiv Temple Complex, Udhampur, Jammu (J\&K), India. Journal of Applied and Natural Sciences 6(2): 816-824.

Ebadati, N., 2017. Statistical Analysis of Dez River Water Quality, Southwest of Iran. Anthropogenic Pollution Journal, 1(1): 46-60.

Gandhi, R. K., Sharma, N., and Raina, A. K., 2017. Ion Chromatographic analysis of heavy metals from a glacial fed cold water Himalayan stream, Bhaderwah, J\&K, India. Environment Conservation Journal, 18(3): 157-165.
Garizi, A. Z., Sheikh, V., and Sadoddin, A., 2011. Assessment of seasonal variations of chemical characteristics in surface water using multivariate statistical methods. International Journal of Environmental Science and Technology, 8(3): 581-592.

Gautam, A., 1990. Ecology and pollution of mountain waster's, Ashish Publishing house, New Delhi.

Giridharan, L., Venugopal, T. and Jayaprakash, M., 2009. Assessment of water quality using chemometric tools: A case study of River Cooum, South India. Archives of Environmental Contamination and Toxicology, 56 (4), 654-669.

Hem, J. D., 1985. Study and Interpretation of the chemical characteristics of the Natural water. U. S. Geological Survey water supply-paper 2254, U. S. Geological Survey, Alexandria.

Jarvie, H. P., Whitton, B. A. and Neal, C., 1998. Nitrogen and phosphorus in east coast British rivers: Speciation, sources, and biological significance. Science of the Total Environment, 210-211, 79-109.

Jha, P. and Barat, S., 2003. Hydrobiological study of lake Mirik in Darjeeling Himalayas. Journal of Environmental Biology, 24(3): 339-344.

Jhingran, V.G.F., 1982. Fish and Fisheries of India. 3rd Edition. Hindustan Publishing Corporation (India), Delhi.

Karthikeyan, K., Nanthakumar, K., Velmurugan, P., Tamilarasi, S. and Lakshmanaperumalsamy, P., 2010. Prevalence of certain inorganic constituents in groundwater samples of Erode district, Tamilnadu, India, with special emphasis on fluoride, fluorosis and its remedial measures. Environmental Monitoring and Assessment, 160(1-4): 141-155.

Khadse, G. K., Patni, P. M. and Labhasetwar, P. K., 2016. Water quality assessment of Chenab River and its tributaries in Jammu and Kashmir (India) based on WQI. Sustainable Water Resource Management, 2(2): 121-126.

Khan, A. A. and Siddqui, A. Q., 1974. Seasonal change in the limnology of a perennial fish pond at Aligarh. Indian Journal of Fishery, 21: 463-478.

Kumar, A. and Singh, A. K., 2002. Ecology, conservation and management of the River Mayurakshi in Santhal Pargana (Jharkhand state) with special reference to effect of sewage pollution on abiotic and biotic potentials. In: Ecology and Conservation and Management of lakes, reservoirs and rivers. Vol. I. (Ed. Arvind Kumar) ABD publishers, Jaipur (India): 1-42.

Majumdar, S. and Dutta, T. K., 2014. Studies on seasonal variations in physico-chemical parameters in Bankura segment of the Dwarakeshwar River (W.B.) India. International Journal of Advanced Research, 2(3): 877881. 


\section{Gandhi et. al.}

Nouri, J., Khorasani, N., Lorestani, B., Karami, M., Hassani, A.H. and Yousefi, N., 2009. Accumulation of heavy metals in soil and uptake by plant species with phytoremediation potential. Environmental Earth Sciences, 59 (2), 315-323.

Nouri, J., Lorestani, B., Yousefi, N., Khorasani, N., Hasani, A. H., Seif, S. and Cheraghi, M., 2011. Phytoremediation potential of native plants grown in the vicinity of Ahangaran lead-zinc mine (Hamedan, Iran). Environmental Earth Sciences, 62(3), 639-644.

Pandey J., Pandey Usha and Tyagi H.R., 2000. Nutrient Status and Cyanobacterial diversity of a tropical fresh water lake. Journal of Environmental Biology, 21(2), 133-138.

Patel, T., Rathod, J., Lakhmapurkar, J. and Gavali, D., 2016. Assessment of Water Quality of Kim River, Gujarat, India. International Research Journal of Natural and Applied Sciences, 4(1): 41-49.

Paudyal, R., Kang, S., Sharma, C. M.,, Tripathee L., Huang J., Rupakheti, D. and Sillanpaa M., 2016. Major ions and trace elements of two selected rivers near Everest region, southern Himalayas, Nepal. Environmental Earth Sciences, 75: 46

Rameez, and Srivastava, S., 2017. Assessment of Water Quality Parameters of Different Sites of River in Srinagar, (Kashmir) India. Archive of life Science and Environment, 1(1): 26-31.

Seenayya, G., 1971. Ecological studies on the plankton of certain freshwater ponds of Hyderabad, (India). II. Phytoplankton. Hydrobiologia, 37: 55-88.

Sekabira, K., Origa, H. O., Basamba, T. A., Mutumba, G. and Kakudidi, E., 2010. Heavy metal assessment and water quality values in urban stream and rain water. International Journal of Environmental Science and technology, 7(4): 759-770.

Semwal, N. and Jangwan, J. S., 2009. Major ion chemistry of River Bhagirathi and River Kosi in the Uttrakhand Himalaya. International Journal of Chemical Sciences, 7(2): 607-616.

Sharma, K. K., Gupta, K., Kouser, U. and Shvetambri., 2015a. Limnological Profile of a High Altitudinal Stream, Bishleri of Banihal Jammu and Kashmir, India. International Journal of Recent Scientific Research, 6(3): 3162-3167.

Sharma, K. K., Singh, D. and Sharma, A., 2015b. Seasonal Variations in the Water Quality Parameters from Manawar
Tawi River in Rajouri District of J\&K, India. Indian Journal of Applied Research, 5(4): 759-763.

Sharma K. K., Sharma A., Singh D. and Gupta S., 2015c. Impact of Pollutants on Water Quality of Basantar River with Reference to Physico-Chemical Parameters of District Samba (J\&K), India. Golden Research Thoughts, 4(12): 111.

Shekhar, C., 1990. Biology of Oreinus richardsonii (Gray and Hard) (From Neeru-Nallah in Bhaderwah) with a view to Formulation of species conservation plan. Ph. D. Thesis submitted to the University of Jammu, Jammu, J\&K, India.

Sivakumar, A. A. and Jaganathan, R., 2002. Hydrology and pollution of the river Bhavani, Tamil Nadu, India. In: Ecology and conservation of lakes, reservoirs and rivers. Vol I (Ed. Arvind Kumar) ABD publishers, Jaipur (India). : 114-131.

Thapa, S., Yonzon, P. B. and Rai, A. K., 2010. Life of a river in the Himalaya: An ecological study of Trisuli River system in Nepal. Aquaculture Asia Magazine (Genetics and Biodiversity), 15(1): 37- 40

Thresh, J. K., Beale, J. F. and Suckling E.V., 1949. The examination of water and water supplies (Ed. E.W. Taylor). London.

Vaishali, P. and Punita, P., 2013. Assessment of seasonal variation in water quality of River Mini, at Sindhrot, Vadodara. International Journal of Environmental Sciences. 3(5): 1424-1436.

Varekar, V., Karmakar, S., Jha, R. and Ghosh, N. C., 2015. Design of sampling locations for river water quality monitoring considering seasonal variation of point and diffuse pollution loads. Environment Monitoring and Assessment, 187: 376.

Wang, X. L., Lu, Y. L., Han, J. Y., He, G. Z. and Wang, T. Y., 2007. Identification of anthropogenic influences on water quality of rivers in Taihu watershed. Journal of Environmental Sciences, 19: 475-48.

Wetzel, R. G., 2001. Limnology; Lake and River Ecosystems, (3rd edition). Academic Press, USA.

WHO, 1999. Guidelines for drinking water quality. World Health Organisation, Geneva.

WHO, 2004. Guideline for Drinking-water Quality. Geneva: volume 1, 3rd edition. World health Organization. 